\title{
Thrombocytosis in Children: Clinical and Heamatological Study in Pediatric Department, Sohag University Hospital
}

\author{
Abd El-Rahman M. Lotfy, Al Zahraa El Sayed Ahmed, Eman M. Fahmy \\ Department of Pediatric, Faculty of Medicine, Sohag University, Sohag, Egypt. \\ *Corresponding author: Abd El-Rahman M. Lotfy, Mobile: (+20) 01090616020, \\ E-Mail: pediatrician.lotfy@gmail.com
}

\begin{abstract}
Background: Thrombocythemia (ET) is defined as elevation in the platelets count more than $400,000 / \mu \mathrm{L}$ in peripheral smear. It is common in infant and children that occurs in 3 to $13 \%$ of children. Thrombocytosis is classified into primary/clonal or secondary according to the pathological origin.

Objective: To determine incidence, causes and types of thrombocytosis in children also the correlations between the severity of thrombocytosis and the etiology and platelets indices.

Patients and methods: Prospective, observational study was done in Pediatric Department and Pediatric Out-Patient Clinic, Sohag University Hospital. A total of 117 cases of thrombocytosis were included in this study. All children had thrombocytosis and aged from one month to twelve years old.

Results: Reactive thrombocytosis was observed in 114 cases (97.44\%), while primary type was found only in 3 cases $(2.56 \%), 2$ of them had chronic myelogenouse leukemia and one case is diagnosed as essential thrombocytosis. Majority of children (96 cases) in our study patients had mild thrombocytosis $(82.05 \%$ ) while moderate thrombocytosis was found in 16 cases and severe thrombocytosis were seen in 5 cases with $13.67 \%$ and $4.27 \%$ of children respectively. The correlation between platelet number and the mean platelet volume (MPV) showed significant negative correlation ( $\mathrm{p}<0.001$ ), while the correlation between the platelet number and plateletcrit (PCT) showed significant positive correlation $(\mathrm{p}<0.001)$ but platelet distribution width (PDW) had no correlation with platelet number $(\mathrm{P}=0.7)$.

Conclusion: Among our study platelet indices, the MPV had a significant negative correlation with platelet count and MPV was considered normal in patients with reactive thrombocytosis.
\end{abstract}

Keywords: Thrombocytosis, Platelet volume, Plateletcrit, Platelet indices.

\section{INTRODUCTION}

Thrombocytosis is defined as increase of platelet count more than $400,000 / \mu \mathrm{L}$ in peripheral smear. It is more common during infancy and childhood and occurs in 3 to $13 \%$ of children ${ }^{(\mathbf{1})}$. Thrombocytosis is classified as primary/clonal or secondary by the pathological origin. Primary thrombocytosis also called essential thrombocythemia is a clonal disease that is exceedingly rare in childhood with the incidence is 1 per million children, which is 60 times lower in children than in adults ${ }^{(2)}$, and may be associated with thromboembolic or hemorrhagic complications. Secondary or reactive thrombocytosis is very common, affects up to $15 \%$ of hospitalized children ${ }^{(3)}$, and occurs secondary to variety of conditions, like acute or chronic infections, iron deficiency anemia, bleeding disorders, hemolytic anemia, collagen vascular diseases, malignancies, drugs or splenectomy ${ }^{(4)}$. Reactive thrombocytosis does not cause any complications and improves spontaneously with treatment of the underlying cause ${ }^{(4)}$. Extreme thrombocytosis (platelets count $>1,000,000 / \mu \mathrm{L}$ ) is less common, occurring in less than $2 \%$ of children, but may occur more common in critically ill children ${ }^{(5)}$.

Primary thrombocytosis is divided into familial and essential. The familial has been described in a number of families and appears to be heterogeneous according to the underlying patho- physiological echanism (6), while essential thrombocytosis, is a chronic clonal myeloproliferative disease ${ }^{(7)}$. Primary thrombocytosis may cause thromboembolic or haemorrhagic complications, and require treatment with platelet lowering agents in some patients according to several factors. Asymptomatic patients need just close follow up and monitoring. Low risk patients need prophylaxis with low dose aspirin. High risk patients need treatment with cytoreductive therapy e.g. hydroxyurea ${ }^{(7)}$. Regarding familial type, familial autosomal recessive, dominant, as well as $\mathrm{X}$ linked forms of primary thrombocytosis have been reported. Spontaneous formation of megakaryopoietic progenitors with increased sensitivity to thrombopoietin (TPO) is found to be the primary mechanisms. In several pedigrees, overproduction of Tpo has been found to be responsible for the disease. In some adult patients mutations in the Tpo gene locus have been found. These mutations typically occur in the 5, untranslated region of the Tpo gene and result in deletions of untranslated open reading frames and overproduction of Tpo. In children with familial thrombocytosis, platelet counts are lower than in ET. Also, splenomegaly is usually not found with almost no thrombotic or hemorrhagic complications occur and prophylaxis is not usually required ${ }^{(\boldsymbol{6})}$.

Hereditary or familial thrombocytosis has been found in several families with varying ethnic origin. Different mutations in the thrombopoietin gene (THPO) have been identified, which affect the upstream open reading frame 7 (uORF 7), which normally has an 
inhibitory effect on TPO translation. Additional cases with various mutations of the cMPL gene have also been found. The mutations identified lead to constitutive MPL activation or secondary increases in TPO due to decreased MPL/TPO binding. More recently, a mutation of JAK2 and JAK2V617I, has been found in a family with hereditary thrombocytosis ${ }^{(\mathbf{8})}$.

The aim of our study was to determine incidence, causes, and types of thrombocytosis also the correlations between etiology, severity of thrombocytosis, and some platelet indices.

\section{SUBJECTS AND METHOD}

Prospective observational study was done in Pediatric Department and Pediatric Out-Patient Clinic, Sohag University Hospital. Children with thrombocytosis from one month age to 12 years old were included in our study. Children with incomplete data were excluded from our study.

All children aged from 1 month to 12 years old attending pediatrics and pediatric super specialty outdoors and indoors in whom thrombocytosis (platelet $>400,000 / \mu \mathrm{L}$ ) were identified during routine blood investigations and were included in the study. Two $\mathrm{mL}$ of EDTA blood was collected through a clean venipuncture from children admitted for whom blood investigations were planned and samples were sent immediately to clinical laboratory and were analysed by the Autoanalizer (ABBOTT cel-Dyn 3700). Once thrombocytosis was observed in the $\mathrm{CBC}$, history taking was done with clear insist on:

-History suggests presence of infection.

-History of anemia.

-History of drug intake e.g., steroid and vincristine.

-History of malnutrition.

-History of systemic inflammatory disorder.

-History of blood transfusion.

-History of splenectomy.

Presenting symptoms and signs with which those children were admitted to the hospital were recorded e.g.: General: pallor, cyanosis, dehydration and lymph node enlargement. Specific: signs of chest infection, organomegaly and arthritis.

Investigations were done to patients with thrombocytosis. $\mathrm{CBC}$ was done and $\mathrm{Hb}, \mathrm{WBC}$ counts, red cell indices, platelet distribution width, mean platelet volume, and platelet CRIT as given by the automated blood cell analyzer for all cases with thrombocytosis.Other relevant investigations, done to establish the diagnosis, were also noted as per the clinical indications in each case. These investigations are such as: CRP, ESR, serum iron and ferritin, bone marrow (BM) biopsy and blood culture.

Etiology of thrombocytosis was obtained from clinical and laboratory data. Correlations between platelet indices, etiology and severity of thrombocytosis were done using SPSS version 24. For the comparison of means and proportions between different groups, unpaired Student's t-test was used. Severity of thrombocytosis was graded according to Dame and Sutor ${ }^{(9)}$ into: (1) Mild $(400,000 / \mu \mathrm{L}-700,000 / \mu \mathrm{L})$. (2) Moderate $(700,000 / \mu \mathrm{L}-900,000 / \mu \mathrm{L})$. (3) Severe (more than $900,000 / \mu \mathrm{L})$. (4) Extreme $(>1000,000 / \mu \mathrm{l})$. Normal values of mean platelet volume (MPV) is 7.4 to $10.4 \mathrm{fL}^{(10)}$, platelet distribution width (PDW) is 10 $17.9 \%{ }^{(11)}$, and plateletcrit (PCT) are 0.17 to $0.34 \%{ }^{(\mathbf{1 2})}$.

\section{Ethical consent:}

An approval of the study was obtained from Sohag University Academic and Ethical Committee. Informed written consent was obtained from parents of all children participants before recruitment in the study, after explaining the objectives of the work. This work has been carried out in accordance with The Code of Ethics of the World Medical Association (Declaration of Helsinki) for studies involving humans.

\section{Statistical analysis}

The collected data were coded, processed and analyzed using the SPSS (Statistical Package for Social Sciences) version 22 for Windows ${ }^{\circledR}$ (IBM SPSS Inc, Chicago, IL, USA). Chi square test $(\chi 2)$ to calculate difference between two or more groups of qualitative variables. Quantitative data were expressed as mean \pm SD (Standard deviation). For the comparison of means and proportions between different groups, unpaired Student's t-test was used. 2-tailed $<0.05$ was considered significant. Also some graphics were performed on GraphPad Prism 9. P $\leq 0.05$ was considered statistically significant.

\section{RESULTS}

A total of 117 cases of thrombocytosis aged from 1 month to 12 years old were included in our study from March 2019 to September 2019. Among these children, 77 cases $(65.81 \%)$ aged from 1 month to 2 years old, 26 cases $(22.22 \%)$ were between 2 - 6 years, and 14 children aged from 6 to 12 years (11.96\%). Thrombocytosis was observed to be more common among boys [ 81 cases, $69.23 \%$ ] than girls [36 cases, $30.76 \%$ ] in all age groups (Figure 1).

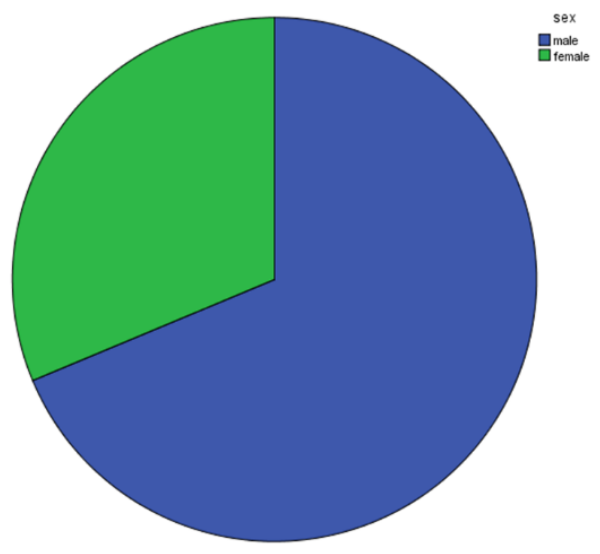

Figure (1): Cases distribution according to sex

Thrombocytosis was classified according to the severity into mild, moderate and severe with platelet 
number 400,000 to $700,000 / \mu \mathrm{L}$ in mild, 700,000 $900,000 / \mu \mathrm{L}$ in moderate and more than $900,000 / \mu \mathrm{L}$ in severe. Majority of children $(n=96)$ had mild thrombocytosis $(82.05 \%)$ while moderate thrombocytosis was found in 16 cases and severe thrombocytosis were seen in 5 cases with $13.67 \%$ and $4.27 \%$ respectively. Among our cases, primary thrombocytosis was found in only three cases (2 CML and 1 essential thrombocytosis) and the remaining (114) patients had secondary thrombocytosis (Table 1).

Regarding reactive thrombocytosis, it was observed that infection (79 cases) was the most common cause associated with secondary thrombocytosis $(67.52 \%)$, followed by anemia (56 cases) with percent of $47.86 \%$. Infection and anemia were found in 29 cases $(24.78 \%)$ having reactive thrombocytosis. Infection alone (without anemia) was found in 50 cases $(42.73 \%$ ) and anemia alone (without infection) was found in 27 cases $(23.07 \%)$. Platelet count in children with infection and anemia was observed to be higher than platelets in children with infection alone or anemia alone.

Among infections, pulmonary infection (41 cases) was the most having thrombocytosis with percentage (35.04\%). Other sites of infections also had thrombocytosis such as gastrointestinal tract infection, urinary tract infection, hepatobiliary infection and central nervous system infection were $24.78 \%, 4,27 \%$ , $1.7 \%$ and $5.98 \%$ respectively. It was observed that thrombocytosis severity increases with increase in severity of infection guided by increase of total white blood cells count. There was a significant positive correlation between platelet number and total leucocytic count $(\mathrm{p}<0.001)$.

Among all types of anemia, iron deficiency anemia was the most commonly associated with thrombocytosis $(27.35 \%)$ and it was observed that the more the severity of anemia was, the more severe the thrombocytosis was and it was statistically significant $(\mathrm{p}=0.04)$. IDA was followed by hemolytic anemia (9.4\%), which was found in 11 cases, of whom 3 cases $(2.56 \%)$ were diagnosed as beta thalassemia major and 6 cases $(5.12 \%)$ were having G6PD deficiency. Autoimmune hemolytic anemia was found in 2 cases $(1.7 \%)$ of SLE. The remaining 13 cases of anemia included variable causes of thrombocytosis. Physiological anemia was found in 5 cases $(4.27 \%)$ and 4 cases had bone marrow suppression due to septicemia (3.41\%). Also, 2 cases with CML had anemia due to bone marrow infiltration. Reactive thrombocytosis following acute hemorrhage was seen in 2 cases (1.7\%), among them 1 case had motor car accident and the other case had bleeding tendency due to late onset vitamin $\mathrm{k}$ deficiency (Table 1).

Rebound thrombocytosis (after recovery from thrombocytopenia) occurred in 3 cases $(2.56 \%)$ and they were receiving oral steroid therapy for treatment of acute ITP (Table 1).
Table (1): Etiology of thrombocytosis with percentage of each

\begin{tabular}{|l|c|c|}
\hline \multicolumn{1}{|c|}{ Etiology } & $\begin{array}{c}\text { Number of } \\
\text { cases }\end{array}$ & Percent \\
\hline Primary & $\mathbf{3}$ & $\mathbf{2 . 5 6 \%}$ \\
thrombocytosis: & 2 & $1.70 \%$ \\
-CML. & 1 & $0.85 \%$ \\
-Essential & & $\mathbf{9 7 . 4 3 \%}$ \\
hrombocytosis. & $\mathbf{1 1 4}$ & $67.52 \%$ \\
\hline Reactive & 79 & $47.86 \%$ \\
thrombocytosis: & 56 & $27.35 \%$ \\
-Infection. & 32 & $9.40 \%$ \\
-Anemia: & 11 & $1.70 \%$ \\
- Iron deficiency anemia & 2 & $4.27 \%$ \\
- Hemolytic anemia & 5 & $3.41 \%$ \\
- Bleeding & 4 & $1.70 \%$ \\
- Physiological anemia & 2 & $2.56 \%$ \\
- BM suppression & 3 & $0.85 \%$ \\
- BM infiltration & 1 & -Drugs (steroid). \\
- Idiopathic. & &
\end{tabular}

In the present study, fever (63.24\%) was the predominant presenting symptom followed by respiratory symptoms like cough and respiratory distress (35.04\%), and GIT symptoms such as vomiting and diarrhea (26.49\%). Regarding signs found in cases of thrombocytosis in our study, pallor $(47.8 \%)$ was the most commonly observed sign followed by signs of chest infection e.g., crepitation and wheezes $(35.04 \%)$ and then signs of dehydration (31.62\%) respectively. In our study, no thromboembolic or hemorrhagic complications were observed (Table 2).

Table (2): Percents of the presenting symptoms

\begin{tabular}{|l|c|c|}
\hline \multicolumn{1}{|c|}{$\begin{array}{c}\text { Presenting } \\
\text { symptoms }\end{array}$} & $\begin{array}{c}\text { Cases } \\
\text { number }\end{array}$ & Percent \\
\hline Fever & 74 & $63.24 \%$ \\
\hline Respiratory & 41 & $35.04 \%$ \\
\hline GIT & 31 & $26.49 \%$ \\
\hline Jaundice & 11 & $9.4 \%$ \\
\hline Urinary & 11 & $9.4 \%$ \\
\hline Neurological & 12 & $10.25 \%$ \\
\hline Skin rash & 6 & $5.12 \%$ \\
\hline Arthritis & 5 & $4.27 \%$ \\
\hline
\end{tabular}

\section{Correlation between severity of thrombocytosis and platelet indices (Table 3):}

Mean of MPV of cases with mild thrombocytosis was $8.51 \mathrm{fL}$, moderate thrombocytosis was $7.38 \mathrm{fL}$, and severe thrombocytosis was $7.33 \mathrm{fL}$. With increasing platelet count, there was a decrease in mean platelet volume and there was a significant negative correlation $(\mathrm{P}<0.001)$ (Figure 2). 


\section{correlation between MPV and PLt}

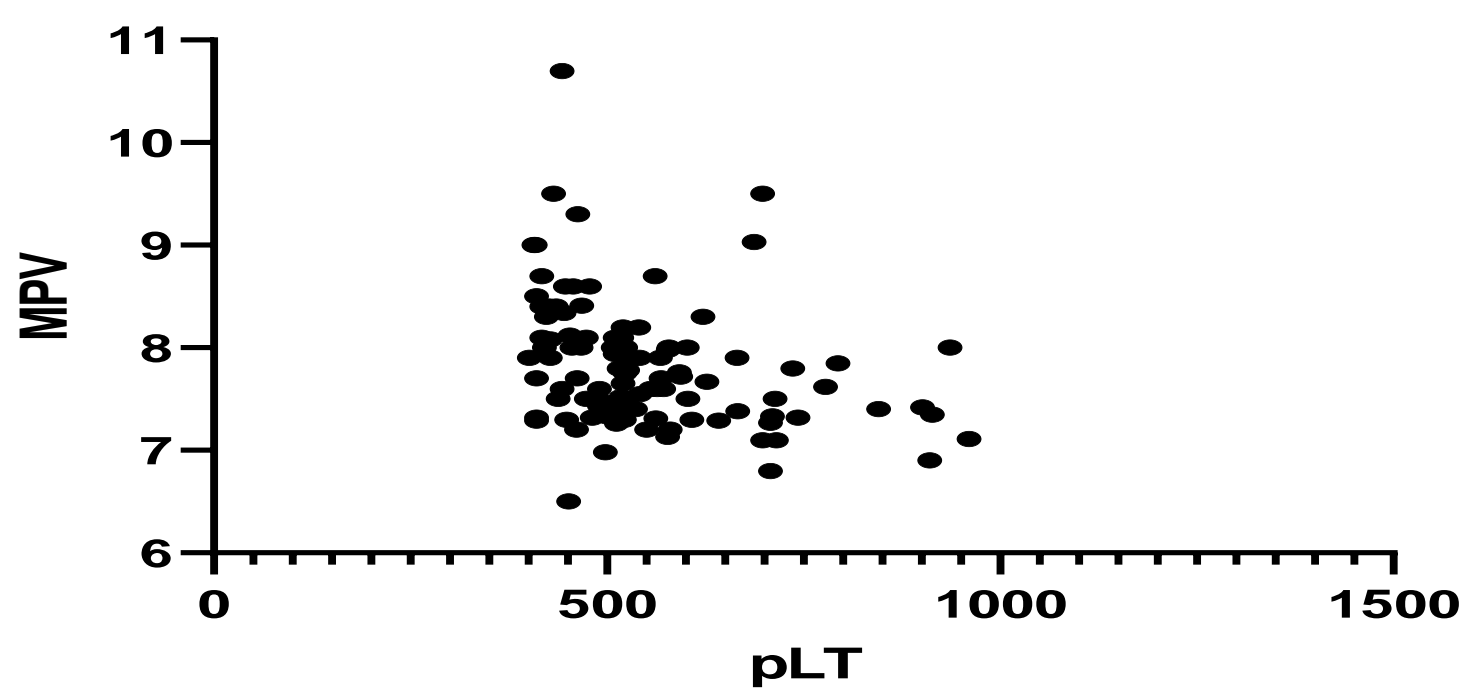

Figure (2): Correlation between MPV and PLT

Mean PDW of children with mild thrombocytosis was $15.63 \%$ and those with moderate and severe thrombocytosis were $16.07 \%$ and $15.50 \%$, respectively. There was no statistical correlation between severity of thrombocytosis and PDW $(\mathrm{P}=0.7)$.

Table (3): Correlation between platelet number and platelet indices

\begin{tabular}{|l|c|c|}
\hline & MPV $($ Mean \pm SD) & PDEW $($ Mean \pm SD $)$ \\
\hline Mild $(\mathrm{n}=96)$ & 8.516 .252 & 15.633 .227 \\
\hline Moderate $(\mathrm{n}=16)$ & 7.380 .323 & 16.071 .657 \\
\hline Severe $(\mathrm{n}-5)$ & 7.3360 .4855 & 15.501 .119 \\
\hline \multicolumn{1}{|c|}{ P value } & $<\mathbf{0 . 0 0 1}$ & $\mathbf{0 . 7}$ \\
\hline
\end{tabular}

Plateletcrit value (PCT) had a significant positive correlation with platelet number (Figure 3).

The more the severity of thrombocytosis was, the more increase in PCT was noticed $(\mathrm{p}<0.001)$.

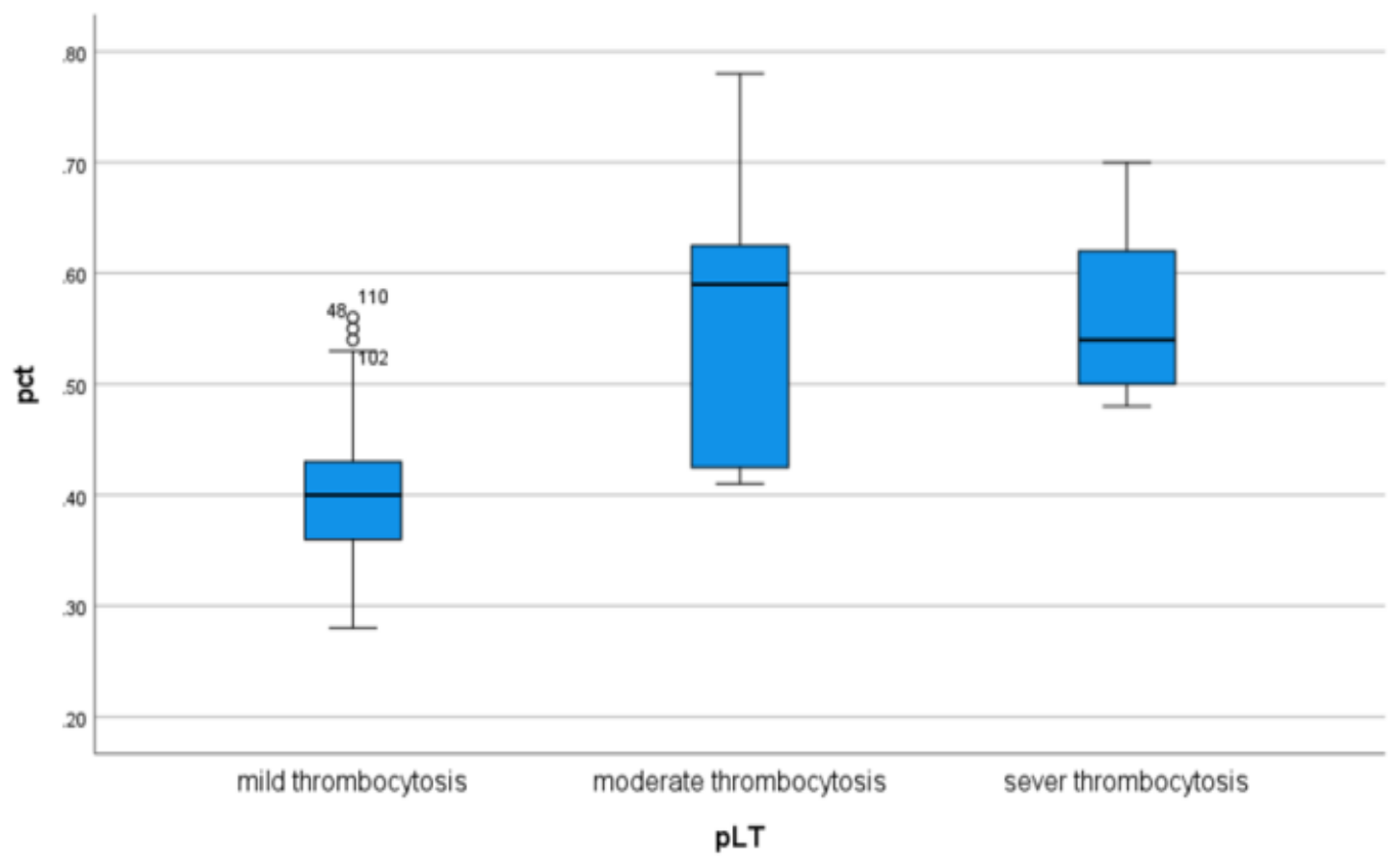

Figure (3): Correlation between severity of thrombocytosis and PCT 


\section{Correlation between severity of thrombocytosis and etiology:}

- In our study, the 3 cases of primary thrombocytosis were associated with severe elevation of platelet count (more than $900,000 / \mu \mathrm{L}$ ), while most cases $(96$ cases) of secondary thrombocytosis were associated with mild thrombocytosis.

- Platelet count in children with infection and anemia was observed to be higher than platelets in children with infection alone or anemia alone.
- It was observed that thrombocytosis severity increases with increase in severity of infection guided by increase of total leucocytic count. There was a significant positive correlation between platelet number and total leucocytic count $(\mathrm{p}<$ 0.001).

- It was, also observed a significant negative correlation between platelet number and hemoglobin level $(\mathrm{p}=0.04)$. The more was the anemia severity, the more thrombocytosis was noticed (Figure 4).

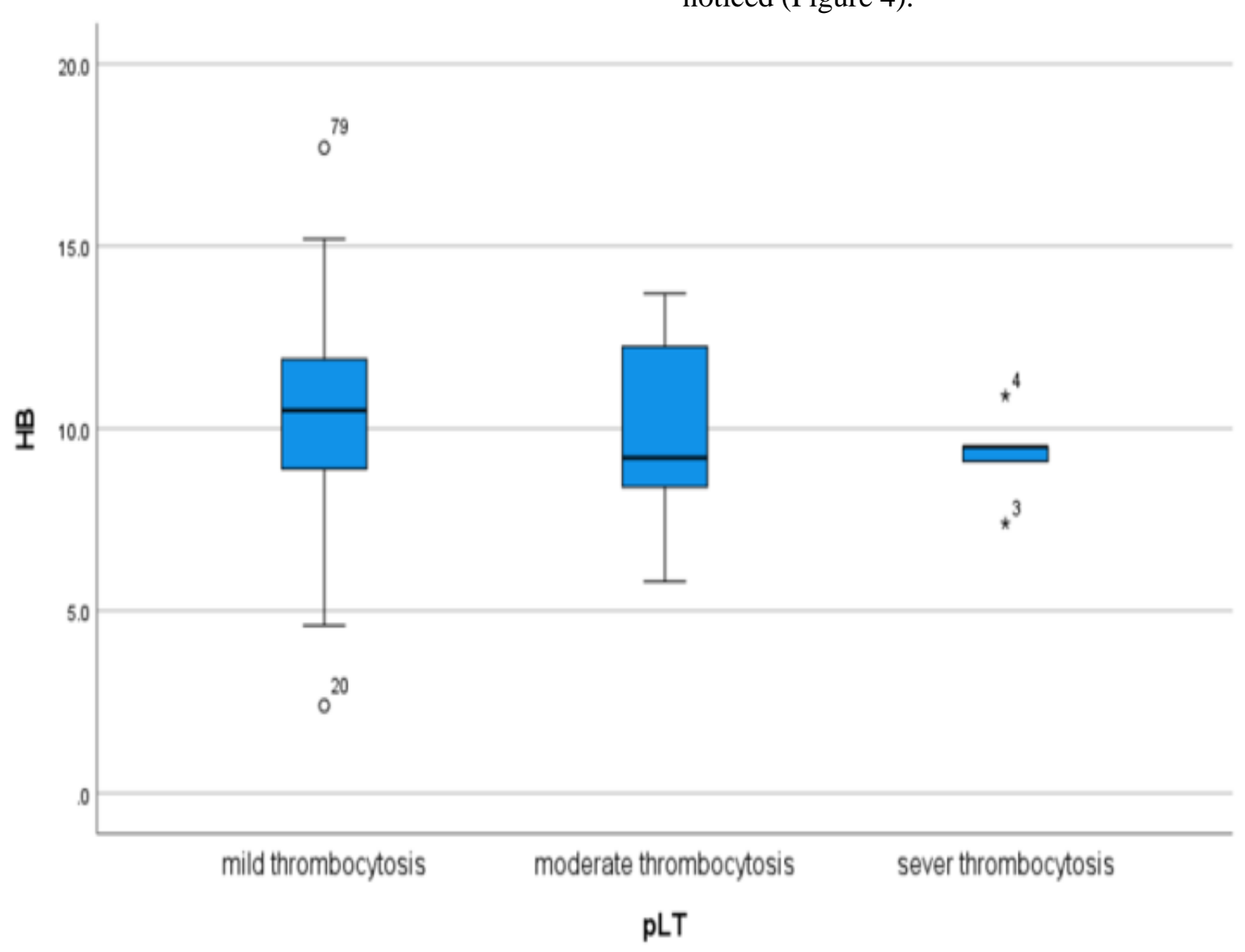

Figure (4): Correlation between platelet number and hemoglobin level.

Table (4): Correlation between platelet indices and etiology

\begin{tabular}{|l|c|c|c|}
\hline Etiology N & Mean MPV & Mean PDW & Mean PCT \\
\hline Primary 3 & 7.866 & 15.60 & 0.55 \\
\hline Secondary 114 & 8.37 & 15.67 & 0.41 \\
\hline
\end{tabular}

- Mean MPV and PDW in primary thrombocytosis were observed to be less than MPV and PDW in secondary thrombocytosis, but no significant results were obtained by T-test. 2-tailed significance of MPV was 0.8, and of PDW was 0.9 .

- Mean PCT for primary thrombocytosis cases was 0.55 , and for secondary thrombocytosis cases was 0.41 . This is due to the positive correlation between platelet number and PCT.

All cases of primary thrombocytosis had severe elevation of platelet count unlike cases of secondary thrombocytosis, most of them have mild platelet elevation. 2-tailed significance was 0.009 (spastically significant).

- MPV was found to have a near significant negative correlation with increase in total leucocytic count $(\mathrm{p}=0.059)$, but PDW has no significant correlation with white blood cell count.

Regarding hemoglobin level, there is a non-significant positive correlation with MPV $(\mathrm{p}=0.3)$, and with PDW $(\mathrm{p}=$ $0.15)$ (Table 4). 


\section{DISCUSSION}

Our study showed that $97.43 \%$ of the studied children had reactive thrombocytosis and $2.56 \%$ only had primary thrombocytosis. Among the only 3 cases with primary thrombocytosis, 2 cases were diagnosed as chronic myelogenouse leukemia (CML) and one case was diagnosed as essential thrombocytosis. All of them had severe thrombocytosis at presentation and had no thrombotic symptoms. In a study done by Subramaniam et al. ${ }^{(13)}, 99.8 \%$ of the study group had reactive thrombocytosis and only two cases have primary thrombocytosis. Among the two children with primary thrombocytosis, one child was diagnosed with chronic myeloid leukemia (CML) and the other child with acute myeloid leukemia (M7). Both of the children had no thromboembolic or hemorrhagic symptoms. Another study done by Yadav et al. ${ }^{(14)}$ showed that among 250 cases, 3 cases only had primary thrombocytosis.

The incidence of reactive thrombocytosis in children differs according to age group. In our study the highest incidence of thrombocytosis has been found in infants aged up to 24 months and after this age the incidence of thrombocytosis decreases ${ }^{(15)}$. The same findings were observed in a study done by Yadav $\boldsymbol{e t} \boldsymbol{a l}$. (14). Thrombocytosis was found to be more common in infants and children aged less than $2 \mathrm{yrs}$ of age $(60 \%)^{(\mathbf{1 5})}$. The higher susceptibility for thrombocytosis during the neonatal period may result from various physiological phenomena: a high Tpo gene expression in the bone marrow during the ontogeny of medullary haematopoiesis, higher circulating Tpo concentrations in fetuses and neonates than in children and adults, and an increased sensitivity of megakaryocyte progenitor cells to Tpo ${ }^{(3)}$.

In our study, thrombocytosis was noticed to be more common among boys (69.23\%) than girls $(30.76 \%)$ in all age groups. Similar results are observed in two studies Yadav et al. ${ }^{(14)}$, and Yohannan et al. ${ }^{(16)}$ with male preponderance of $64 \%$ and $61.1 \%$ of cases, respectively. Testosterone has synergistic effect on thrombopoiesis. Clinical and molecular evidence suggests that sex hormones specifically androgens mediate platelet count and function ${ }^{(17)}$.

Severity of thrombocytosis was divided into 3 grades mild, moderate and severe according to platelet number as mentioned before. In our study mild thrombocytosis $(82.05 \%)$ was more commonly than moderate (13.67\%) and severe (4.27\%) thrombocytosis. Among children with severe thrombocytosis the maximum platelet count observed was $950,000 / \mu \mathrm{L}$ in a baby 10 months old with gastroenteritis, severe dehydration and bronchopneumonia associated with severe iron deficiency anemia. Similar observations are seen in the study of Yadav et al. ${ }^{(14)}$ in which mild thrombocytosis was most commonly observed.

In this study, fever was the predominant presenting symptom $(63.24 \%)$ followed by respiratory symptoms like cough and respiratory distress (35.04\%) while GIT symptoms such as vomiting and diarrhea were found in $(26.49 \%)$. Jaundice was found in 11 cases (9.4\%), 6 of them had acute hemolysis, 3 cases are known to have beta thalassemia major and 2 cases had acute hepatitis A virus infection.

Convulsion was present in 12 cases (10.25\%), 8 of them had central nervous system infection, 2 cases had febrile convulsion, 1 case had intracranial hemorrhage due to late onset vitamin K deficiency and the last case developed convulsion due to brain hypoxia after attack of acute severe hemolytic anemia.

Pallor was the most commonly founded sign (47.8\%) followed by signs of chest infection like fine crepitations and wheezes (35.04\%) and signs of dehydration were founded in $31.62 \%$. Arthritis was observed in 5 cases, 3 of them had HSP and 2 cases were diagnosed as SLE.

In the study done by Subramaniam et al. ${ }^{(13)}$, fever was the predominant presenting symptom $(50.1 \%)$ followed by respiratory symptoms like cough (35\%) and respiratory distress (8.8\%). Pallor was the most commonly observed sign (30\%) followed by hepatomegaly $(18.5 \%)$, splenomegaly $(6.4 \%)$, and hepatosplenomegaly (13.5\%).

In our study, infection in different sites was the most common cause of secondary thrombocytosis 79 cases $(67.52 \%)$ among them 29 cases $(24.78 \%)$ were associated with anemia and 50 cases had infection alone (42.73\%). Similar results are obtained by Heng and Tan ${ }^{(18)}$, and Chan et al. ${ }^{\left({ }^{19)}\right.}$ studies in which 105 cases (78\%) and 37 cases (37\%) had reactive thrombocytosis due to infection respectively.

Respiratory system infection was the most common findings (35.04\%) followed by GIT infection $(26.49 \%)$. In the study done by Subramaniam et al. ${ }^{(13)}$, infection with anemia was most commonly associated with secondary thrombocytosis $(48.3 \%)$, which is not similar to our study. This difference could be due to less number of cases studied in our study in comparison with

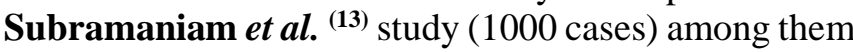
483 cases had infection and anemia and 162 cases secondary thrombocytosis was due to intercurrent infection alone and anemia (without infection) was present in 172 patients. Among infections, respiratory tract infections $(n=283)$ were most commonly associated with thrombocytosis (28.3\%).

In our study, platelet count in children with infection and anemia was observed to be higher than platelets in children with infection alone or anemia alone. It was observed that thrombocytosis severity increases with increase in severity of infection guided by increase of total leucocytic count. There was a significant positive correlation between platelet number and total leucocytic count $(\mathrm{p}<0.001)$.

Regarding non-infectious causes of reactive thrombocytosis, iron deficiency anemia was the second cause in our study after infections, since it is the single most common nutritional deficiency worldwide (20). Thrombocytosis is more frequent in children up to 2 
years of age is partly due to the higher incidence of iron deficiency in this age group and the more the severity of anemia was, the more severe the thrombocytosis ${ }^{(\mathbf{1})}$.

Hemolysis also is a common cause of thrombocytosis. In our study 11 cases $(9.4 \%)$ had hemolytic anemia. Acute hemolysis was present in 6 cases and their diagnosis was G6PD deficiency and 3 cases had chronic hemolytic anemia and diagnosed as beta thalassemia major. Autoimmune hemolytic anemia was noted in 2 cases of SLE. In two other studies done by Yohannan et al. ${ }^{(16)}$ and Chan et al. ${ }^{(19)}$, hemolytic anemia was observed in 128 cases (19.3\%), and 22 cases (22\%) respectively.

It was also observed that increase in anemia severity was associated with more increase in the platelet number and it was statistically significant ( $\mathrm{p}=$ 0.04), and this is similar to Subramaniam et al. ${ }^{(13)}$ study. Corticosteroids are known to cause transient thrombocytosis as a result of release of stored platelets from the spleen into the blood circulation ${ }^{(21)}$. In our study, 3 cases were receiving oral steroid therapy for treatment of acute ITP and showed rebound thrombocytosis after one week of steroid intake. In a study done by Yohannan et al. ${ }^{(16)}, 3$ cases with ITP developed rebound thrombocytosis following steroid treatment. In ITP, megakaryopoiesis increased in response to immune-mediated destruction of platelets. Activated platelets tend to aggregate leading to excessive clotting or thromboembolic complications. During the treatment, platelet overproduction in the phase of decreased or normalized platelet destruction may result in thrombocytosis ${ }^{(22)}$.

In our study, mean MPV and PDW in primary thrombocytosis were observed to be less than MPV and PDW in secondary thrombocytosis. Raised PDW and MPV may be suspicious for clonal disease ${ }^{(\mathbf{1 2})}$, but this is not found in our study, which may be due to smaller number of children studied here unlike Subramaniam et al. ${ }^{(13)}$.

\section{CONCLUSION}

Thrombocytosis is common laboratory finding in pediatric that occurs commonly in males than females. In most cases thrombocytosis is reactive type and most commonly founded in boys less than 2 years of age.

Primary thrombocytosis, a clonal disease that is extremely rare and may be complicated with thromboembolic or haemorrhagic disorders. Some patients having ET may need treatment with platelet lowering therapy according to several factors. Asymptomatic patients need just close follow up and monitoring. Low risk patients need prophylaxis with low dose aspirin. High risk patients need treatment with cytoreductive therapy e.g., hydroxyurea.

Severe thrombocytosis was detected in children having primary thrombocytosis more than in those with reactive thrombocytosis.
Among our study on platelet indices, the MPV has a significant negative correlation with platelet count and the mean MPV was considered normal in patients with reactive thrombocytosis.

\section{REFERENCES}

1. Mantadakis E, Tsalkidis A, Chatzimichael A (2008): Thrombocytosis in childhood. Indian Pediatrics, 45: 669- 677.

2. Hasle $\mathbf{H}$ (2000): Incidence of essential thrombocythaemia in children. Br J Haematol., 110: 751-55.

3. Dame C (2002): Developmental biology of thrombopoietin in the human fetus and neonate. Acta Paediatrica., 91: 54-65.

4. Harrison C, Bareford D, Buttetal N (2010): Guide line for investigation and management of adultsand children presenting with a thrombocytosis. British Journal of Haematology, 149: 352-375.

5. Denton A, Davis $P$ (2007): Extreme thrombocytosis in admissions to paediatric intensive care: no requirement for treatment. Arch Dis Child, 92: 515-516.

6. Fujiwara T, Harigae H, Kameoka J et al. (2004): A case of familial thrombocytosis: possible role of altered thrombopoietin production. Am J Hematol., 76: 395397.

7. Kucine N, Chastain K, Mahler M et al. (2014): Primary thrombocytosis in children. Haematologica, 99 (4): 620-8.

8. Teofili L, Larocca $L$ (2011): Advances in understanding of the pathogenesis of famililalthrombocythaemia. Br J Haematol., 152 (6): 701-12.

9. Dame C, Sutor A (2005): Primary and secondary thrombocytosis in childhood. Br J Haematol., 129: 165-177.

10. Demirin H, Ozhan H, Ucgun T et al. (2011): Normal range of mean platelet volume in healthy subjects: Insight from a large epidemiologic study. Thromb Res., 128 (4): 358-60.

11. Farias M, Schunck E, Dal Bó S et al. (2010): Definition of reference ranges for the platelet distribution width (PDW): a local need, Clinical Chemistry and Laboratory Medicine, 48: 255-257.

12. Giovanetti T, do Nascimento A, de Paula J (2011): Platelet indices: laboratory and clinical applications. Rev Bras Hematol Hemoter., 33 (2): 164-5.

13. Subramaniam N, Mundkur S, Kini $P$ et al. (2014): Clinicohematological study of thrombocytosis in children. ISRN Hematol., 2014:389257.

14. Yadav D, Chandra J, Sharma S et al. (2010): Clinicohematological study of thrombocytosis. Indian Journal of Pediatrics, 77: 643-647.

15. Matsubara K, Fukaya T, Nigami H et al. (2004): Agedependent changes in the incidence and etiology of childhood thrombocytosis. Acta Haematologica, 111: 132-137.

16. Yohannan M, Higgy K, Al-Mashhadani S et al. (1994): Thrombocytosis. Etiologic analysis of 663 patients. Clin Pediatr., 33: 340343-48

17. Li A, Karlan B (2005): Androgen mediation of thrombocytosis in epithelial ovarian cancer biology. Clinical Cancer Research, 11: 8015-8018.

18. Heng J, Tan A (1998): Thrombocytosis in childhood. Singapore Med J., 39: 485-487.

19. Chan K, Kaikov Y, Wadsworth L (1989): Thrombocytosis in childhood: a survey of 94 patients. Pediatrics, 84: 1064-1067.

20. Sandoval C (2002): Thrombocytosis in children with iron deficiency anemia: series of 42 children. Journal of Pediatric Hematology/Oncology, 24: 593.

21. Lin C, Yang Y, Lee C et al. (2006): Thrombopoietin and interleukin-6 levels in Henoch-Schoenleinpurpura. J Microbiol Immunol Infect., 39: 476-482.

22. Pietra D, Li S, Brisci A et al. (2008): Somatic mutations of JAK2 exon 12 in patients with JAK2 (V617F) negative myeloproliferative disorders. Blood, 111 (3): 1686-9. 